BULLETIN OF THE

AMERICAN MATHEMATICAL SOCIETY

Volume 78, Number 1, January 1972

\title{
SIGNATURES ON SEMILOCAL RINGS
}

\section{BY MANFRED KNEBUSCH, ALEX ROSENBERG ${ }^{1}$ AND ROGER WARE $^{2}$}

Communicated July 28, 1971

We announce extensions of a part of the Artin-Schreier theory of real fields to semilocal rings. Detailed proofs will appear elsewhere.

$A$ always denotes a (not necessarily noetherian) semilocal commutative ring such that no residue class field has only two elements. A signature on $A$ is a homomorphism $\sigma$ from the unit group, $A^{*}$, of $A$ to $\{ \pm 1\}$ with $\sigma(-1)$ $=-1$ and $\sigma\left(l^{2}+a m^{2}\right)=1$ for all triples $(a, l, m)$ in $A^{*} \times A \times A$ such that $l^{2}+a m^{2}$ is a unit and $\sigma(a)=1$.

EXAMPLES. (i) If $A$ is an integral domain and $<$ is a total ordering of $A$ then $\sigma: A^{*} \rightarrow\{ \pm 1\}$ defined by $\sigma(a)=1$ if $a>0$ and $\sigma(a)=-1$ if $a<0$ is a signature. If $A$ is a field the signatures correspond bijectively with the orderings of $A$.

(ii) Let $A$ be the local ring of the affine curve $X^{2}+Y^{2}=0$ over the real field $\boldsymbol{R}$ at $(0,0)$. Then the signature

$$
\sigma: A^{*} \stackrel{v}{\rightarrow} R^{*} \stackrel{s}{\rightarrow}\{ \pm 1\}
$$

obtained by composing the evaluation map $v$ at $(0,0)$ and the unique signature $s$ of $\boldsymbol{R}$ does not arise from an ordering of $A$.

(iii) For valuation rings we are able to analyze the situation to some extent : Let $A$ be a valuation ring with maximal ideal $\mathrm{m}$. Then any signature $\sigma$ arises from an ordering of $A$. If $A$ has rank one and $\sigma(1+\mathfrak{m}) \neq 1$, then $\sigma$ arises from a unique ordering. If $A$ is a discrete rank one valuation ring and $\sigma(1+\mathfrak{m})=1$, then there are exactly two orderings on $A$ inducing the signature $\sigma$.

Let $A$ now again be a general semilocal ring as above.

Proposition 1. Let $\sigma$ be a signature, $a_{1}, \ldots, a_{r}$ units of $A$, and $l_{1}, \ldots, l_{r}$ elements of $A$ such that $b=l_{1}^{2} a_{1}+\cdots+l_{r}^{2} a_{r}$ is also a unit. Then $\sigma\left(a_{1}\right)$ $=\cdots=\sigma\left(a_{r}\right)=1$ implies $\sigma(b)=1$.

DEFINITION. A subset $M$ of $A^{*}$ is saturated if $M$ is a subgroup of $A^{*}$ and $a_{1}, \ldots, a_{r}$ in $M$ implies $b$ in $M$ for all units $b=l_{1}^{2} a_{1}+\cdots+l_{r}^{2} a_{r}$ with $l_{i}$ in $A$.

Thus Proposition 1 states that for any signature $\sigma$ the set

$$
\Gamma(\sigma)=\left\{a \text { in } A^{*} \mid \sigma(a)=1\right\}
$$

is saturated.

AMS 1970 subject classifications. Primary 15A63, 13H99; Secondary 06A70, 12J15.

1 Partially supported by NSF Grant GP-25600.

2 Partially supported by NSF Grant GP-28915. 
Definition. For any subset $M$ of $A^{*}$ the saturation $\hat{M}$ is the smallest saturated set containing $M$.

The elements of $\hat{M}$ are easily seen to be the units of the form

$$
b=\sum_{0 \leqq i_{j} \leqq 1} c_{(i)} a_{1}^{i_{1}} a_{2}^{i_{2}} \cdots a_{r}^{i_{r}}
$$

with $a_{1}, \ldots, a_{r}$ in $M$ and the $c_{(i)}$ sums of squares in $A$.

Theorem 2 (CF. [12, SATZ 21 and 1, SATZ 2]). Assume 2 is in $A^{*}$. Then, for any subset $M$ of $A^{*}$,

$$
\hat{M}=\bigcap \Gamma(\sigma),
$$

where $\sigma$ runs through the set of signatures with $\Gamma(\sigma) \supset M$.

Theorem 2 for $A$ a field is due in this generality to E. Witt (unpublished). We next state three special cases of Theorem 2 :

(1) The $\Gamma(\sigma)$ are the maximal saturated proper subsets of $A^{*}$.

(2) Let $M=\{1\}$. The units $b$ of $A$ with $\sigma(b)=1$ for all signatures $\sigma$ are precisely the units which are sums of squares (cf. [1, Satz 1]). In particular -1 is a sum of squares if and only if $A$ has no signatures (cf. [2, Satz 7b]).

(3) Let $A^{\prime}$ be a semilocal subring of $A$ without any residue class fields containing only two elements. A signature $\sigma$ of $A^{\prime}$ can be extended to a signature of $A$ if and only if there is no equation $-1=l_{1}^{2} b_{1}+\cdots+l_{r}^{2} b_{r}$ with $b_{i}$ in $A^{\prime *}, \sigma\left(b_{i}\right)=1$ and $l_{i}$ in $A$ (cf. [4, Theorem 1, p. 34]).

It has been shown by one of us [8] that in a local ring $A$ with -1 a sum of squares, the least number of squares needed to represent -1 is of the form $2^{n}$ if 2 is in $A^{*}$ and of the form $2^{n}$ or $2^{n}-1$ otherwise.

Let $B$ a finite etale extension of $A$, in other words $B$ is a finitely generated projective $A$-module and a projective $B \otimes_{A} B$-module [6, Proposition 18.3.1, p. 114]. To generalize the Artin-Schreier theory further it seems to be important to study extensions of signatures of $A$ to those of $B$. We have only obtained partial results concerning this problem:

Proposition 3. Let $\sigma$ be a signature of $A$ and assume for simplicity that the projective $A$-module $B$ is free of rank $[B: A]$.

(i) If $[B: A]$ is odd then $\sigma$ has at least one extension to $B$.

(ii) Suppose $B=A[X] /(f(X))$ with $f(X)$ a monic polynomial in $A[X]$. (This is always the case if $B$ is local.) If there exists some $c$ in $A$ such that $f(c)$ is a unit in $A$ with $\sigma(f(c))=-1$, then $\sigma$ has at least one extension to $B$ (cf. [4, Proposition 3, p. 36]).

(iii) If $B$ is a galois extension of $A$ as defined in $[3]$ and [5], then $\sigma$ has either no extension to $B$ or exactly $[B: A]$ extensions.

(iv) Assume $[B: A]=2$, so that $B=A[X] /(f(X))$ with $f(X)=X^{2}-X$ $-c$ for an element $c$ in $A$ such that $1+4 c$ lies in $A^{*}$. Then $\sigma$ has an extension to $B$ if and only if $\sigma(1+4 c)=1$. 
In contrast to Artin-Schreier, we strongly use the structure theorems for the Witt ring, $W(A)$, of free nondegenerate symmetric bilinear spaces over $A[\mathbf{9}],[\mathbf{1 0}]$ to prove these results. The connection arises from the following theorem:

THEOREM 4 (CF. [7] AND [11]). The signatures of A correspond bijectively with the ring homomorphisms $W(A) \rightarrow Z$. The correspondence associates to each signature $\sigma$ a homomorphism $\psi_{\sigma}$ mapping a space (a) of rank one to $\sigma(a)$.

D. K. Harrison had already suggested that Proposition 3(iii) could be proved for galois field extensions of odd degree by considering the Witt ring without making use of the theory of real closures $[14, \S 5]$.

Every subset $M$ of $A^{*}$ yields an ideal a $(M)$ of $W(A)$ generated by the binary spaces $(1,-a)$ with $a$ in $M$. Results on multiplicative forms [8] imply:

THEOREM 5. Let 2 be in $A^{*}$. Then for any subset $M$ of $A^{*}$ the radical of $\mathfrak{a}(M)$ is $\mathfrak{a}(\hat{M})$.

EXAMPLE. Considering $M=\{1\}$ we obtain: If 2 is in $A^{*}$, the $\operatorname{ring} W(A)$ has no nonzero nilpotent elements if and only if every unit of $A$ which is a sum of squares is a square itself (cf. [11]).

\section{REFERENCES}

1. E. Artin, Über die Zerlegung definiter Funktionen in Quadrate, Abh. Math. Sem. Univ. Hamburg 5 (1927), 100-115.

2. E. Artin and O. Schreier, Algebraische Konstruktion reeler Körper, Abh. Math. Sem. Univ. Hamburg 5 (1926), 85-99.

3. M. Auslander and O. Goldman, The Brauer group of a commutative ring, Trans. Amer. Math. Soc. 97 (1960), 367-409. MR 22 \# 12130.

4. N. Bourbaki, Algèbre. Chap. 6, 2nd ed., Actualités Sci. Indust., no. 1179, Hermann, Paris, 1964.

5. S. U. Chase, D. K. Harrison and A. Rosenberg, Galois theory and Galois cohomology of commutative rings, Mem. Amer. Math. Soc. No. 52 (1965), 15-33. MR 33 \# 4118.

6. A. Grothendieck, Eléments de géométrie algèbrique. IV. Étude locale des schémas et des morphismes de schémas. IV, Inst. Hautes Études Sci. Publ. Math. No. 32 (1967), 361 pp. MR 39 \# 220.

7. D. K. Harrison, Witt rings, Lecture Notes, Department of Mathematics, University of Kentucky, Lexington, Kentucky, 1970.

8. M. Knebusch, Runde Formen über semilokalen Ringen, Math. Ann. 193 (1971), 21-34.

9. M. Knebusch, A. Rosenberg and R. Ware, Structure of Witt rings, quotients of abelian group rings, and orderings of fields, Bull. Amer. Math. Soc. 77 (1971), 205-210.

10. - Structure of Witt rings and quotients of abelian group rings, Amer. J. Math. (to appear).

11. F. Lorenz und J. Leicht, Die Primideale des Wittschen Ringes, Invent. Math. 10 (1970), $82-88$.

12. A. Pfister, Quadratische Formen in beliebigen Körpern, Invent. Math. 1 (1966), 116-132. MR 34 \#169.

13. - Zur Darstellung von -1 als Summe von Quadraten in einem Körper, J. London Math. Soc. 40 (1965), 159-165. MR 31 \# 169.

14. A. Rosenberg and $\mathrm{R}$. Ware, The zero-dimensional galois cohomology of Witt rings, Invent. Math. 11 (1970), 65-72.

Mathematisches Institut, Universität des SaArlandes, D-660C SaARbrücken, GERMANY

Department of Mathematics, Cornell University, Ithaca, New York 14850

Department of MATHEMatics, Northwestern University, EVANSTON, Illinois 60201 